Bioética

\section{Prevenção DA TRANSMISSÃO VERTICAL DO HIV}

Recentemente, o Conselho Regional de Medicina do Estado de São Paulo, considerando "a gravidade da infecção pelo HIV e AIDS no Brasil... destacandose a crescente feminização da epidemia", bem como "as altas taxas de transmissão vertical do HIV no país, apesar da disponibilização de tratamento gratuitos às gestantes na rede pública, incluindo o acesso aos medicamentos anti-retrovirais" e, finalmente, "a comprovada eficácia do tratamento anti-retroviral durante a gravidez, o trabalho de parto e as primeiras semanas de vida, o que permite redução significativa do risco de um recém-nascido contrair o vírus HIV de uma gestante soropositiva", editou a seguinte Resolução':

Artigo ${ }^{\circ}$ - É dever do médico solicitar à mulher, durante $o$ acompanhamento pré-natal, a realização do exame para detecção do HIV, com aconselhamento pré e pós-teste, resguardado o sigilo profissional.

Artigo $2^{\circ}$ - É dever do médico fazer constar no prontuário médico a informação de que o exame anti-HIV foi solicitado, bem como o consentimento ou a negativa da mulher em realizar o exame.

Artigo $3^{\circ}$ - Os serviços e instituições de saúde, públicos e privados, devem proporcionar condições para o exercício profissional, disponibilizando exames, medicamentos e outros procedimentos necessários ao diagnóstico e tratamento da infecção pelo HIV em gestantes, bem como assistência ao pré-natal, parto, puerpério e atendimento ao recémnascido.

\section{Comentário}

A transmissão do vírus da imunoficiência humana (HIV) da mãe para a criança determina uma infecção crônica e fatal, que agora pode ser prevenida com a utilização de adequada terapêutica anti-retroviral; os índices de transmissão mãe-criança, que sem tratamento são superiores a $20 \%$, podem ser reduzidos a $2 \%$ ou menos, quando a carga viral materna é diminuída a níveis indetectáveis por terapêutica anti-retroviral agressiva, ou quando a profilaxia com zidovudina é combinada com a realização de cesariana eletiva². Por isso, a Resolução do CREMESPé importante: não é admissível, hoje, que os médicos deixem de fazer o diagnóstico de infecção materna e de adotar condutas terapêuticas adequadas, capazes de impedir quase completamente a transmissão vertical do HIV.

Resoluções dos Conselhos de Medicina, por si só, não mudam a conduta dos médicos; seu papel educativo, porém, é indiscutível.

\section{Gabriel Wolf Oselka}

\section{Referências}

I. Resolução CREMESP n 95, de 14/ I I/00, publicada no Diário Oficial do Estado, secção I, n 223, de 22/I I/00. p. 48.

2. Mofenson LM. Perinatal expusure to zidovudine - benefits and risks. N Engl J Med 2000; 343: 803-5.

\section{Clínica Médica \\ Cetoacidose DIABÉTICA: CONSIDERAÇÕES SOBRE 0 TRATAMENTO}

AAmerican Diabetes Association propõe dois esquemas de insulinoterapia para o tratamento da cetoacidose diabética, um deles usando insulina endovenosa (EV) na dose de $0, \mathrm{I}$ unidade / $\mathrm{kg}$ peso/hora com ajuste conforme resposta da glicemia: dobrando a taxa de infusão quando a glicemia diminuir em velocidade menor que 50-70 $\mathrm{mg} / \mathrm{dl} /$ hora e diminuindo esta infusão quando a queda da glicemia for maior que $70 \mathrm{mg} /$ dl. Outro esquema adotado pela mesma Associação preconiza o uso de insulina regular IM ou subcutânea (SC): bolo inicial de 0,4 unidades $/ \mathrm{Kg}$ (metade dessa dose inicial emboloEVemetadevialMouSC) edepois mantendo dose de $0, \mathrm{I}$ unidade/Kg/hora IM ou SC, observando a taxa de queda da glicemia, que deve ser mantida entre $50 \mathrm{e}$ $70 \mathrm{mg} / \mathrm{dl} /$ hora. Quando a glicemia chegar a níveis menores que $250 \mathrm{mg} / \mathrm{dl}$, o $\mathrm{pH}$ arterial for maior que 7,30, o bicarbonato arterial tiver valores maiores que $18 \mathrm{mEq} / \mathrm{l}$ e os corpos cetônicos diminuírem, considera-se que ocorreu a reversão do quadro de cetoacidose e o controle passa a ser realizado com insulina Regular (SC) de 4 em 4 horas conforme glicemia capilar, ea prescrição do dia seguinte utilizará insulina NPH com 2/3 da dose total de insulina regular no dia anterior ou de $0,6 / \mathrm{U} / \mathrm{Kg}$ de peso. A reposição de potássio é importante, pois o 
potássio corporal total estará diminuído; entretanto, devido à acidose, o nível de potássio pode estar aumentado no meio intravascular, portantoa reposição só deve ser realizada se sua concentração sérica estiver dentro danormalidade.

\section{Comentário}

No Pronto Socorro do Hospital das Clínicas/FMUSP utilizamos insulina regular IMdevido à eficácia comparável ao uso de insulina EV e pelo maior cuidado que a terapia endovenosa exige na monitorização do paciente, cuidados estes nem sempre possíveis de serem realizados em uma unidade de emergência. $O$ tratamento adequadonormalmente reverte a condição em tempo médio de 4 a 7 horas. O cuidado na velocidade de diminuição das taxas de glicose no sangue e a hidratação com observação clínica cuidadosa tendem a evitar as complicações do tratamento e explicam porque a mortalidade da condição declinou nos últimos anos.

Rodrigo antonio Brandão neto Augusto Scalabrini Neto

\section{Referências}

I. Alberti KG, Hockaday TD, Turner RC. Small doses of intramuscular insulin in the treatment of diabetic coma. Lancet 1973: 515-522.

2. KitabchiAE, Umpierrez GE, Murphy MB, etal. Managementof hyperglycemic crises in patients with diabetes mellitus (Technical Review). Diabetes Care 200I; 24:I3I-53. 3. American Diabetes Association: Hyperglicemic crises in patients with diabetes mellitus. Diabetes Care 200 I; 24: I54-6I.

4. Morris LR, Murphy MB, Kitabchi AE. Bicarbonate therapy in severe diabetic ketoacidosis. Ann Int Med 1986;105:836-40.

\section{Clinica Ciriongiea}

\section{Videotoracoscopia NO TRAUMA DE TÓRAX}

Oempregodavideocirurgianodiagnóstico etratamentodostraumatismostorácicosconstituiumcampoaberto. Poucosãoos relatos de suaaplicaçãoe, portanto, apadronizaçãotécnica esuaindicaçãonãoestão bemestabelecidas'

A condição fundamental para indicação da videotoracoscopia no trauma torácicoé a estabilidade hemodinâmica. Os doentes instáveis evidentemente requerem um tratamento imediato, condição que exclui qualquer tipo de exame, inclusive a videotoracospia. Portanto, doentes estáveis com hemotórax ou hemo-pneumotórax, ferimentos toracoabdominais, ferimentos transfixantes do mediastino ou então portadores de corpos estranhos intrapleurais, constituem os eventuais grupos que poderão beneficiar-se da videotoracoscopia.

Avideocirurgiaédefundamentalimportância para o diagnóstico delesões do diafragma, nosferimentos datransiçãotoracoabdominal em doentesassintomáticos. Semestemétodo diagnóstico, nestes casos, a lesão passará despercebida.

Osobjetivosdesteprocedimentosão:diagnóstico precocedeumalesão, fontedesangramento ou de escape de ar eo seutratamento, sema realização da toracotomia, evitando-se assimum traumatismomaior paraodoente.

A experiência do Serviço de Emergência da Santa Casa de São Paulo, com 52 doentes, permite-nos concluir que 0 procedimento é eficaz e apresenta pouca ou nenhuma morbidade 2 .

\section{Comentário}

Comodesenvolvimento davideotoracoscopia, o emprego de toracotomias para diagnóstico de doença pleuropulmonar, especialmente de nódulos solitários, diminui consideravelmente.

Entreasgrandes vantagens dessemétodo estãoa poucaagressividade, apossibilidadede diagnósticoprecisoeterapêuticaadequadasem toracotomianoscasoseletivosenasurgências.

ÉaexperiênciadoServiçodeEmergênciada Santa Casa de São Paulo que nos permitiu aquilatarseuvalorem váriasoportunidades.

Roberto SAAd JÚNIOR

Referências

I. Saad Jr Re Rasslan S. A videocirurgia no trauma de tórax. Rev Col Bras Cir 1993; 20:1II.

2. Dorgan Neto, V Saad Jr, R Rasslan S. Videotoracoscopiano trauma de tórax. Rev Col Bras Cir 200I ; 28:3-8.

\section{Economia da Saide

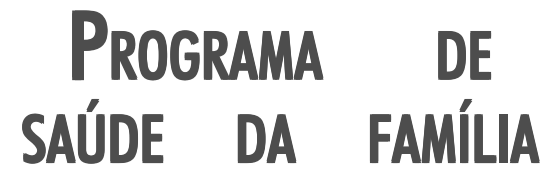

O Programa de Saúde da Família (PSF) de 1994, desenvolvido a partir do Programa de Agentes Comunitários de Saúde (PACS) de 1991, vem causando alterações significativas na alocação de recursos, forma de pagamento de serviços emodelo de assistência médica. OPACS e o PSF apresentam uma discriminação positiva em favor da população carente no país, vêm atuando na prevenção e promoção da saúde e estão provocando a antecipação da demanda e toda uma reorganização dos serviços de saúde. 
Cada grupo do PSF, composto por médico, enfermeiro, auxiliar de enfermagem e 4 a 6 agentes comunitários, atinge em média cerca de 800 a 1000 famílias e recebe de $R \$ 28.000,00$ a $R \$$ $54.000,00$ do SUS por ano (além de R\$ I.500,00 por agente comunitário)'. Em junho de 2000, o PACS cobria mais de 26 milhões de brasileiros e o PSF quase 10 milhões. Mas a distribuição geográfica do PACS/PSF ainda é bastante diferenciada. Alagoas, Ceará e Minas Gerais, responsáveis por $50 \%$ da população coberta pelo PSF no Brasil em junho de 2000, já apresentavam um grau de cobertura do PSF respectivamente de $31 \%$, 22\% e $13 \%$ da população em cada um dos estados ${ }^{2}$.

A importância crescente do PACS/ PSF pode ser avaliada pela evolução da participação destes programas nos recursos do SUS. O PACS/PSF, que representavam $4 \%$ do total dos recursos destinados para o Atendimento Ambulatorial e para a Atenção Básica em 1998, atingiram 12\% em 2000. ${ }^{2}$

\section{Comentário}

OPSF está sendo apresentado como um modelo de assistência à saúde para atingir a população brasileira carente $e$ de alto risco. Mais da metade da população brasileira apresentava renda familiar per capita inferior a I salário mínimo e $76 \%$ não estavam cobertas por planos privados de saúde em 1998. ${ }^{3}$ O PSF certamente tornará possível racionalizar os gastos públicos com saúde e, desta forma, ampliar e melhorar a assistência médica no Brasil.

Em recente debate, o coordenador do Curso de Especialização em Saúde da Família da USP, Rubens Kon, o exsecretário nacional de Assistência à Saúde, Gilson Carvalho, e a médica de família Monique Bourget discutiram os avanços e desafios do PSF. Os debatedores acreditam que o PSF conseguirá diminuir a demanda por especialistas e racionalizar o uso da tecnologia, das referências, da hospitalização, etc. Entretanto, a racionalização dos custos, a princípio, não deverá ser acompanhada pela redução do custo da saúde porque quando se dá atenção primária, automaticamente abre-se uma porta para tudo que está acumulado. "Pode ser que racionalizando o custo se consiga atender todo mundo. Mas, para fazer atenção primária de verdade, o custo não vaiser menor...", afirma Monique Bourget ${ }^{4}$.

Samuel Kilsztajn

\section{Referências}

I. Viana ALD, Lima LD. Family health program in Brazil. Rio de Janeiro, Instituto de Medicina Social/UERJ, 200I (texto apresentado e distribuído no General Population Conference, 24, 200 I, Salvador - mimeo).

2. Ministério da Saúde. Informações de saúde. Brasília, MS, 200I. Disponível: http://tabnet.datasus.gov.br/tabnet/ tabnet.htm.

3. Kilsztajn S, Camara MB, Carmo MSN. Gasto privado com saúde por classes de renda. In: IUSSP. General Population Conference, 24, 200I, Salvador. Anais de Demografia Brasileira... Campinas: ABEP, 200 I. CD-ROM I6p. Disponível: http://www.abep.org.br.

4. Médico de família. Ser médico (CRMSP), 200I; 4: 20-8.

\section{Ginecologia \\ Recomendações DA AMERICAN HEART SOBRE DE HORMONAL NO CLIMATÉRIO E PREVENÇÃO SECUNDÁRIA}

Adecisão de se utilizara terapia dereposição com estrogênio (TRE) após a menopausa, objetivandoa prevençãosecundária da doençacardiovascular(DCV)tempropiciado inúmeras polêmicas. Isto ocorreu principalmente após a publicação do HERS (Heart Estrogen ReplacementStudy)que, diferentemente dos estudos observacionais prévios, mostrou que usuárias acima de 65 anos, da associação estrogênio conjugado eqüino (ECE, na dose de $0,625 \mathrm{mg} /$ dia) com acetato demedroxi-progesterona (AMP, na dose de 2,5mg/dia), com história de infarto do miocardio, revascularização coronariana ou evidências angiográficas decoronariopatia, apresentavam aumento da mortalidade, quando comparadoaoplacebo. Esse incrementosignificativodamortalidadeocorriano primeiroano deusodoshormônios enodecorrer dosanos seguintes reduzia-se, porém, de forma não significativa. Emfunçãodisso, aAmerican Heart Association (AHA) resolveu se posicionar, emitindo um "Statementfor HealthcareProfessionals" comasseguintes recomendações: I)a TRH não deve ser iniciada paraa preven- 
ção secundária; 2) a decisão para continuar ou suspender a TRH em mulheres com $\mathrm{DCV}$, que já estavam em uso por longo tempo, deve se basear nos benefícios e riscos estabelecidos não-coronarianos e na preferência da paciente; 3) caso uma muIher desenvolva um evento agudo ou necessite permanecer imobilizada na vigência da TRH, é prudente que se descontinue o uso da TRH ou que se considere a profilaxia do tromboembolismo venoso. A reintrodução da TRH deve se basear nos benefícios eriscos estabelecidosnão-coronarianos, bem como na preferência da paciente.

\section{Comentário}

OHERS, apesarde serumestudo prospectivo, randomizado e controlado com placebo, apresenta algumas limitações. Entre elas, citam-se: I) a população estudada eracompostademulheresidosas (acimade 65 anos) e não-climatéricas; 2) o hipolipemiantefoimais prescritono grupo placebo, o que poderia explicar a menor mortalidade, em relação ao grupo usuário de hormônios; 3) a dose de estrogênio prescrita foi considerada alta para este grupo etário; 4) a adição do progestogênio poderia reduzir os efeitos cardioprotetores dos estrogênios e 5) o estrogênio usado por via oral propiciou o maior risco de tromboembolismo, que de fato ocorreu no primeiro ano de uso do hormônio. Entendemos que o "Statement" daAHA éoportuno e deve ser seguido; entretanto, é fundamental que se considere as limitações do HERS e que novos estudos devam ser realizados com outros tipos de esteróides, com doses distintas e por outras vias com o intuito de melhor aclarar esta questão.

José Mendes Aldrighi antonio de Pádua Mansur Claudia Maria Santos Aldrighi
Referência

Mosca L, Collis P, Herrington DM, et al. Hormone replacement therapy and cardiovascular disease. A statement for healthcareprofessionalsfrom the American Heart Association. Circulation. 200 I; 104:499-503.

\section{Obstetricia}

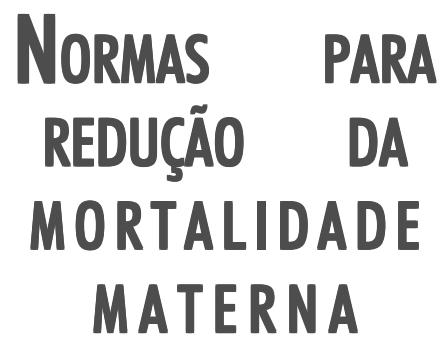

A mortalidade materna, no contexto mundial, éutilizada como parâmetro parase avaliar a qualidade do serviço de saúde ofertado a uma população. Em outras palavras, quanto menor for a razão de mortalidade materna (RMM) de uma localidade, melhor a qualidade de saúde disponibilizada. A RMM declarada para o Brasil em 1998 foi de 65,8 óbitos maternos por 100.000 nascidos vivos. Porém, somente I I das 27 capitais (incluído o Distrito Federal) possuem Comitês de Mortalidade Maternaatuantes ou em fase de implantação, e apenas o Comitê do Paraná executa um trabalho que abrange todo o Estado. Isso significa dizer que esta razão é bem maior do que a divulgada. Na cidade de São Paulo, onde existe um dos Comitês de Mortalidade Materna mais atuantes, verifica-se uma subnotificação do óbito materno em torno de $50 \%$ a $60 \%$ '. Em um país de dimensões continentais, com realidades distintas, éimportante a existência de Comitês de Mortalidade Materna com a função de identificar os problemas regionais e estabelecer medidas apropriadas para saná-los. Para uma redução adequada da RMMé imprescindível a implantação de medidas básicas de saúde, como um planejamento familiar bem estruturado, um pré-natal consciente ecoerente, com drenagem de casos patológicos para serviços de alto-risco. Estes serviços devem garantir uma referência para o parto, em hospitais equipados para tal atendimento eamparados por berçários de qualidade. Deve-se evitar a alta do pré-natal, acompanhando-se agestante semanalmente até a vigência do parto. Os hospitais que atendemas gestantes sem patologia devem proceder auma admissão supervisionada e mais coerente, visando a redução da peregrinação da gestante por vários serviços ${ }^{2}, 0$ que geralmente ocasiona a transformação de um caso normal em um caso complicado. $O$ atendimento à gestante deve ser regionalizado, evitando-se grandes deslocamentos para a realização da consulta de pré-natal e do parto.

\section{Comentário}

Oavanços tecnológicos naárea da saúdenão param de acontecer. Éfundamental uma reciclagem constante da equipe multiprofissional que assessora agestante, para que o atendimento seja feito de forma digna, amparada por conhecimentos e tecnologia de ponta. As normas sugeridas visam o atendimento da população mais carente e, à primeira vista, parecem simples de serem executadas. Dependem, entretanto, de uma enorme vontade política agregada a uma mobilização da classe médica como um todo, para que realmente surtam o efeito desejado. Como se isto não bastasse, a nutrição da nossa população é deficiente e mal-orientada, com certeza predispondo ao agravamento das doenças. Diminuir a mortalidade materna é um trabalho árduo e sem resultados 
imediatos, porém de suma importância para o bem-estar de nossa população.

Carlos eduardo Pereira Vega

Referências

I. Pazero LC, Marcus PAF, Vega CEP, Boyaciyan K, Barbosa SA. Estudo da mortalidade materna no Município de São Paulo durante o ano de 1996. Rev Bras Ginecol Obstet 1998, 20:395-403.

2. Tanaka ACA. Maternidade: dilema entre nascimento e morte. São Paulo: HucitecABRASCO, 1995.

\section{Pediatria}

\section{HorMôNIO DO CRESCIMENTO - PARTE I}

A Sociedade de Pesquisa em Hormônio de Crescimento reuniu-se para avaliar alguns aspectos do uso do hormônio $(\mathrm{GH})$ e verificar se algumas preocupações com sua utilização encontram respaldo na literatura especializada.

Ao lado da análise sobre a segurança de seu uso em crianças, a Sociedade debruçou-se sobre os aspectos de segurança com o uso do $\mathrm{GH}$ em adultos.

Metabolismo da glicose-A prevalência de diabetes mellitus (DM) está aumentada em adultos hipopituitários e as ações metabólicas do GH incluem antagonismoà insulina. Recomenda-se que o metabolismo glicêmico sejaavaliado em todos os pacientes, antes e após substituição com GH. DM ou tolerância a glicose alterada não são contra-indicações do uso de GH. O cuidado de diabéticos em uso de $\mathrm{GH}$ deveseguir as recomendações usuais, com monitorização intensificada no início do tratamento. $O$ exame de fundo de olho deve ser periodicamente realizado e, se bem que uma retinopatia não-proliferativa não seja motivo de descontinuação do uso de $\mathrm{GH}, \mathrm{O}$ desenvolvimento de retinopatia proliferativa exige a suspensão do uso do hormônio decrescimento.

Retenção de fluidos - Pode ocorrer, especialmente nas fases iniciais do uso do GH. Parcialmente, isto refletea normalização da hidratação tecidual, induzida por $\mathrm{GH}$ edependente de dose. Peso, história clínica e exame físico são capazes de identificar tal ocorrência. Redução de dose no caso de sintomas persistentes deve ser considerada. Cuidado especial em pacientes com insuficiência cardíaca congestiva.

Interação com outros hormônios- 0 $\mathrm{GH}$ aumenta a conversão de T4 a T3 e pode desmascarar um hipotireoidismo incipiente. Todos os pacientes devem ser monitorizados quanto àfunção tireoideana. $\mathrm{O} \mathrm{GH}$ pode diminuir a concentração de cortisol total diminuindo a globulina ligadora (CBG). Pode também reduzir a biodisponibilidade de cortisol, aumentando sua conversão a cortisona. A possibilidade de uma insuficiênciaadrenal deveser considerada durante a terapia.

Função cardíaca e lipoproteínas - A prevalência aumentada de doença cardiovascular observada na acromegalia ativa não pode ser extrapolada para o adulto hipopituitário em reposição de GH. Dessa forma, a monitorização da função cardiovascular deve seguir os padrões para população normal. A substituição de $\mathrm{GH}$ no adulto aumenta os níveis séricos de lipoproteínas. São incertas as implicações (seé que há) dessas elevações e isto deve ser pesado contra os benefícios da instituição do tratamento com GH. Não recomendamosas dosagens de lipoproteínas como um procedimento rotineiro em pacientes hipopituitários.

Risco de câncere recorrência de tumor - tem sido documentada uma incidência aumentada de certas doenças malignas(especialmente recorrência de tumor) em adultos hipopituitários, mas não há evidência que tal fato associe-seà reposição com $\mathrm{GH}$. As recomendações usuais para prevenção de câncer e detecção precoce devem ser implementadas nos pacientes recebendo $\mathrm{GH}$.

Aspectos de segurança em tratamento farmacológico de GHemadultos-Uso de Terapia Intensiva-Estudos demonstram que a mortalidade dobra em pacientes gravemente enfermos tratados com altas doses de GH. Em dois estudos controlados por placebo, envolvendo 522 pacientes, demonstrou-se que a mortalidade aumentou de $19 \%$ para $42 \%$ no grupo que recebeu $\mathrm{GH}$.

Tratamentosfarmacológicos com $\mathrm{GH}$ Qualquer tratamentocom $\mathrm{GH}$ que não seja de substituição em pacientes deficientes deve ser considerado farmacológico. Em condições específicas em que se esteja considerando o tratamento farmacológico, devem ser coletados dados de segurança e protocolos devem ser desenvolvidos, como se estivéssemos testando uma nova droga. A evolução negativa observada em pacientes em terapia intensiva que receberam altas doses de GH não podem ser automaticamente extrapolados para outras condições que podem, eventualmente, beneficiar-se deste tipo de uso.

\section{Comentário}

Cada vez mais tem havido indicações para o uso de GHem pacientes adultos que 
foram crianças que cresceram normalmentee, por conseqüência, produziram seu $G H$ nessa fase de crescimento. Os cuidados a serem tomados em tais pacientes são bem abordados pela Sociedade de Pesquisa em $\mathrm{GH}$ e abertas quanto a doses farmacológicas devem ser tomados em sua devida conta. Alguns "mitos" têm sidoderrubados, como a contra-indicação do GH em casos deneoplasiase, extremamente importante, o seguimento dos pacientes, com avaliações periódicas como intuito de se detectar alterações que podem levarà suspensão da medicação(como retinopatia proliferativa, por exemplo) nunca deve ser esquecido.

\section{Durval Damiani}

\section{Referência}

Growth Hormone Research Society. Critical evaluation of the safety of recombinant human Growth Hormone administration: statement from the Growth Hormone Research Society. J. Clin Endocrinol Metab, 200I; 86: 1868-70.

\section{Medicina Baseada em Evidêneias} Diagnóstico E CONDUTA NA RUPTURA TRAUMÁTICA DA
AORTA TORÁCICA

A ruptura traumática da aorta é responsável por cerca de 8.000 mortes por ano nos Estados Unidos, causadas principalmente por acidentes automobilísticos, atropelamentos, quedas e esmagamentos torácicos. Agrande maioria dos pacientes tem morte instantânea. Os $10 \%$ al $5 \%$ que chegam vivos ao hospital podem sobreviver se a lesão for diagnosticada e tratada rápida e eficazmente. Como nenhum centro de trauma possui enorme experiência no tratamento destas lesões, as diretrizes propostas são baseadas nas evidências atualmente disponíveis.

Nivelldeevidência: Comonãoháestudo clínico prospectivo, randomizado, não dispomos de nível I de evidência para estabelecer uma conduta padrão.

Nivel Ildeevidência: As recomendações listadas a seguir são baseadas em estudos prospectivos não-comparativos, ou retrospectivos com dados confiáveis: I) a possibilidade de lesão traumática da aorta deveser considerada em todos os pacientes envolvidos em colisões automobilísticas, independente da direção do impacto; 2) a radiografia de tórax é um bom exame de triagem, determinando a necessidade de investigações adicionais; os achados mais significantes incluemalargamento do mediastino, botão aórtico pouco definido, desvio do brônquio fonte esquerdo ou de sonda nasogástrica e opacificação da janela aortopulmonar; 3) a aortografia tem alta sensibilidade, especificidade e acuidade, sendo considerada o exame-padrão contra qual as outras modalidades devem ser comparadas; 4) tomografia computadorizada é um testeútil, tanto como triagem como para o diagnóstico dalesão; a técnica helicoidal ou espiral tem valor preditivo negativo extremamente elevadoe pode ser usada isoladamente para excluir lesão da aorta, reservando-sea angiografia para exames duvidosos; 5) o reparo imediato da lesão é preferível quando o paciente tem outras lesões com risco imediato de vida, tais como anecessidade de craniotomia ou laparotomia urgente, ou o paciente é de alto risco cirúrgico, por idade avançada ou comorbidades, 0 reparo deveser retardado. Nestes casos, o controlemedicamentoso da pressão arterialdeve ser realizado até que a cirurgia possa ser realizada.

Nívelllldeevidência:Asseguintesrecomendações são baseadas em séries de casos retrospectivasouem revisão de bancodedados: I) a presença de pseudo coartação ou sopro interescapular devedesencadear investigação subseqüente; 2) oeco transesogágico étambémum exame sensível e específico, porém requerdisponibilidade, treinamentoeexperiência do examinador; 3) o reparo da lesãoé melhor conduzido com alguma técnica de perfusão distal; complicações neurológicas, principalmentea paraplegia, têm correlação com o tempo de isquemia que, portanto, deve ser o menor possível.

\section{Comentário}

Umalto índice de suspeita, baseadono mecanismo de trauma, é a maneira de rapidamente realizar odiagnóstico e tratamento desta lesão de alta letalidade. As novas técnicas multislice, que permitem tomografias de excelente qualidade em segundos, são altamente promissores. Em virtude da raridade dos pacientes que chegam vivos, o papel das técnicas de perfusão distal deve ser determinado em estudos multicêntricos prospectivos. Enquanto isso, o rápido avanço das técnicas de reparo com próteses endovasculares provavelmenteassumirão papel prioritário no tratamento destas lesões.

Luiz francisco Poli de figueiredo Ruy Jorge Cruz JR

Referência

Guidelinesfor the diagnosisandmanagement ofbluntaorticinjury. Eastern Association for the Surgery of Trauma, 2000. Disponível: www.east.org. 